\title{
On the Practical Feasibility of Secure Distributed Computing
}

A Case Study

\author{
GREGORY NEVEN, FRANK PIESSENS*, BART DE DECKER \\ Department of Computer Science, K. U. Leuven \\ Celestijnenlaan 200A, B-3001 Leuven, Belgium \\ \{gneven,frank,bart\}@cs.kuleuven.ac.be
}

Keywords: Secure distributed computing, cryptography, communication overhead

\begin{abstract}
Secure Distributed Computing addresses the problem of performing a computation with a number of mutually distrustful participants, in such a way that each of the participants has only limited access to the information needed for doing the computation. Over the past decade, a number of solutions for this problem have been developed. The various proposed solutions differ in the cryptographic primitives that are used, and in the class of computations that can be performed. However, all sufficiently general solutions have one thing in common: the communication overhead between the involved parties seems to be prohibitive.

In this paper, we consider a concrete instance (with considerable practical interest) of the general problem of secure distributed computing, and we investigate how bad the communication overhead really is. This involves tailoring the different general solutions to the specific problem at hand, optimizing them for minimal communication overhead, and evaluating the resulting communication overhead.
\end{abstract}

\section{INTRODUCTION}

Secure distributed computing addresses the problem of distributed computing where some of the algorithms and data that are used in the computation must remain private. Usually, the problem is stated as follows, emphasizing privacy of data. Let $f$ be a publicly known function taking $n$ inputs, and suppose there are $n$ parties (named $p_{i}, i=1 \ldots n$ ),

*Postdoctoral Fellow of the Belgian National Fund for Scientific Research (F.W.O.)

The original version of this chapter was revised: The copyright line was incorrect. This has been corrected. The Erratum to this chapter is available at DOI: 10.1007/978-0-387-35515-3_53 
each holding one private input $x_{i}$. The $n$ parties want to compute the value $f\left(x_{1}, \ldots, x_{n}\right)$ without leaking any information about their private inputs (except of course the information about $x_{i}$ that is implicitly present in the function result) to the other parties. An example is voting: the function $f$ is addition, and the private inputs represent yes $\left(x_{i}=1\right)$ or no $\left(x_{i}=0\right)$ votes. In case you want to keep an algorithm private, instead of just data, you can make $f$ an interpreter for some (simple) programming language, and you let one of the $x_{i}$ be an encoding of a program. In descriptions of solutions to the secure distributed computing problem, the function $f$ is usually encoded as a boolean circuit, and therefore secure distributed computing is also often referred to as secure circuit evaluation.

An efficient solution to the secure distributed computing problem would be an enabling technology for a large number of interesting distributed applications across the Internet. Some example applications are: auctions without a trusted third party, charging for the use of algorithms on the basis of a usage count ([8]), various kinds of weighted voting, protecting mobile code integrity and privacy ([7]), ...

Over the past decade, a large variety of solutions to the problem has been proposed. An overview is given by Franklin [4]. These solutions differ from each other in the cryptographic primitives that are used, and in the computations that can be performed (some of the solutions only allow for specific kinds of functions to be computed). However, these ingenious, powerful solutions have not yet been used in practical applications, mainly because they incur a very large communication overhead.

Given the fact that communication bandwidth increases steadily, and research on more efficient solutions to the secure distributed computing problem slowly decreases the communication overhead, it is clear that practical feasibility of these protocols is only a matter of time. In this paper, we investigate how close we are to practical applicability of secure computing technology. In section 2 , we describe a concrete problem of practical interest that requires this technology. Next, we try to solve the problem in the most efficient way, using various techniques for secure distributed computing proposed in the literature. Finally, we conclude by assessing the acceptability of the communication overhead.

\section{AN EXAMPLE APPLICATION}

In this section, an interesting application that requires the use of secure distributed computing technology is introduced, and a number of assumptions about this application (like estimations of the size of the inputs to the application) are made explicit. This application could be 
implemented based on conventional cryptography and a trusted third party, but if it must be implemented without trusted third party, the use of secure distributed computing techniques is essential.

Suppose Alice is human resource manager of a company looking for new employees and Bob makes a living gathering CVs in a database and selling these to interested companies. Bob's database contains a wide variety of records but Alice is not willing to pay for a CV she's not interested in: she only wants records that satisfy a certain query. Of course, Bob doesn't want to reveal his entire database if Alice is only going to buy a few records from it, but on the other hand Alice might want to keep her query secret from Bob (maybe she doesn't want her competitors to know about her selection criteria). What they need is a so-called Secret Query Database, that works as follows.

Let's denote Alice's query as $q$ and one of Bob's records as $x$. This Secret Query Database must allow them to cooperate in such a way that they can compute $q(x)$ while Alice preserves the secrecy of $q$ and Bob preserves that of $x$. The result of $q(x)$ is a single bit: a one if $x$ satisfies $q$ or a zero if it doesn't. In case the outcome of $q(x)$ is a one, Bob sends the record to Alice and Alice pays for it. In case it is a zero, Bob keeps the record for himself.

What exactly is meant by secrecy of the query is a fine matter. In this paper, we try to achieve the following level of secrecy: in the assumption that the query is completely random, that $E$ is the set of already evaluated records (so Bob knows $q(x)$ for every $x \in E$ ) and that $y \notin E$, Bob should not be able to predict $q(y)$ non-negligibly better than random guessing. This definition allows Bob to know which records Alice wants, but not why she wants those records. If we look at $q$ as a huge truth table with on every line a possible record as input and the result bit of the query for that record as output, we can say that Bob learns only the lines that correspond to records he evaluated together with Alice, but he can't tell anything about the other lines. A stronger definition would be for Bob to learn only how many records are selected (for payment purposes) but not the exact identity of the records. This would lead us to a generalization of Private Information Retrieval (PIR), introduced in [2], which is a conceptually different problem. Neither has the concept of Secret Query Database anything to do with the problem of statistical inference from aggregate database queries, which is discussed in [3].

No matter which protocol is used, there must always be some minimal interaction. Neither of both parties should be able to evaluate a query without any help from the other. More specifically, after evaluating $q(x)$ together, Bob should not be able to learn $q\left(x^{\prime}\right)$ without help from Alice and in the same way Alice should not be able to calculate $q^{\prime}(x)$ all by 
herself. If the first condition doesn't hold, it is trivial to see that our definition of secrecy of Alice's query is violated. If the second doesn't hold, Alice could subsequently evaluate the queries "is the $i$ th bit of $x$ a one?" for every bit in $x$ and thus obtain the entire record $x$ without paying for it.

Most protocols for secure distributed computing represent the function to be evaluated as a logical circuit. An important issue if we want to estimate the communication complexity is the number of gates and inputs needed in those circuits. With the concrete example of Bob's database containing CVs in mind, we use the following notation: the size of a single record is $i_{s}$ bits or $i_{s} / 8$ bytes. A circuit representing a query takes $C$ gates on the average. Realistic values for the example of the Secret Query Database are: $i_{s}=4000, C=1000$. Some protocols allow one party to hide the circuit from the other parties, but some don't. If we want to use the latter to implement a Secret Query Database, some kind of universal circuit is needed where Bob's secret input is still one of his records but Alice's secret input is an encoding of her query (in other words, as discussed in the introduction, we need an 'interpreter circuit' that can interpret a simple query language). The number of gates of such a universal circuit is denoted as $C_{u}$. It is feasible to construct such a universal circuit allowing most common queries using $C_{u}=4000$ gates. The encoding of a query would take about $i_{c u}=1000$ bits.

Protocols for secure distributed computing typically require a lot of network resources. Sometimes, we can reduce the communication overhead if Bob publishes reusable data on CD-ROM. By reusable, we mean that it must be possible to use this data over and over again in multiple evaluations without leaking any additional information about the records or the query, even if these evaluations are performed with gossiping clients or - which is essentially the same thing - with one and the same client. This method is most useful for slowly evolving databases like the one containing CVs. Bob can then publish this CD-ROM on a regular but not too frequent basis in relatively large quantities.

In the remaining text of this paper, Bob is referred to as the server, while Alice is called the client.

\section{POSSIBLE SOLUTIONS 3.1. USING OBLIVIOUS TRANSFER}

In [6], Goldreich, Micali and Wigderson present a two-party protocol for the problem of combined oblivious transfer which is equivalent to the problem of secure circuit evaluation. The two players $A$ and $B$ proceed as follows. $B$ assigns two random bit strings $r_{i}^{0}$ and $r_{i}^{1}$ to every wire $i$ 
in the circuit, which represent an encoded 0 and 1 on that wire. This defines a mapping $\phi_{i}: r_{i}^{b} \mapsto b$ for every wire $i$. $B$ also chooses a random bit string $R$ that will allow $A$ to check if a decryption key is correct. The general idea of the protocol is that, if $b$ is the bit on wire $i$ in the evaluation of the circuit for $A$ 's and $B$ 's secret inputs, $A$ will only find out about $r_{i}^{b}$ and will never get any information about $\phi_{i}\left(r_{i}^{b}\right)$ or $r_{i}^{\bar{b}}$. In other words, $A$ evaluates the circuit with encoded data.

We use the notation $E(M, r)$ for a symmetric encryption function of the message $M$ with secret key $r$. To encrypt a NOT-gate with input wire $i$ and output wire $o, B$ constructs a random permutation of the tuple $<E\left(R \cdot r_{o}^{1}, r_{i}^{0}\right), E\left(R \cdot r_{o}^{0}, r_{i}^{1}\right)>$ where $\cdot$ denotes the concatenation of bit strings. To encrypt an AND-gate with input wires $l$ and $r$ and output wire $o, B$ constructs a random permutation of the tuple

$<E\left(R \cdot r_{o}^{0}, r_{l}^{0} \oplus r_{r}^{0}\right), E\left(R \cdot r_{o}^{0}, r_{l}^{0} \oplus r_{r}^{1}\right), E\left(R \cdot r_{o}^{0}, r_{l}^{1} \oplus r_{r}^{0}\right), E\left(R \cdot r_{o}^{1}, r_{l}^{1} \oplus r_{r}^{1}\right)>$

with $\oplus$ the bit-wise XOR. Any other binary port can be encrypted in an analogous way.

$B$ sends the encryption of every gate in the circuit together with $R$, the encoding of his own input bits and the mapping $\phi_{m}$ of the output wire $m$ to $A$. To perform the evaluation of the circuit on encoded data, $A$ first needs encodings of all the input bits. For $B$ 's input bits, the encoding was sent to her, but since $B$ doesn't know $A$ 's inputs, $B$ can't send an encoding of them. Note that $B$ can't send the encoding of both a 1 and a 0 on $A$ 's input wires either, because that would allow $A$ to find out more than just the result of the circuit. The technique that is used to get the encoding of $A$ 's input to $A$ is called one-out-of-two oblivious transfer. This is a protocol that allows $A$ to retrieve one of two data items from $B$ in such a way that (1) $A$ gets exactly the one of two items she chose and (2) $B$ doesn't know which item $A$ has got.

Thus, $A$ and $B$ execute a one-out-of-two oblivious bit string transfer (often referred to as $\left(\begin{array}{l}2 \\ 1\end{array}\right)-\mathrm{OT}^{k}$ ) for each of $A$ 's input bits. This guarantees that $A$ only obtains the encoding of her own input bits without releasing any information about her bits to $B$. $A$ evaluates each gate by trying to decrypt every element of the tuple using the encoding of the bit on the input wire (or the XOR of two input bit encodings) as a key; she will only decrypt one of the elements successfully, thereby obtaining the encoded bit on the output wire. Note that she can know if a decryption was correct by comparing the first bits of the decrypted string with $R$. Proceeding this way through the entire circuit, $A$ obtains the encoding of the final output and applies $\phi_{m}$ to reveal the plain output bit.

How can this protocol fit in an implementation of a Secret Query Database? The circuit is visible to both parties (it is not an ambition 
of the protocol to hide the circuit), so we need a universal circuit. The server plays the role of $B$, the circuit scrambler, and the client takes $A$ 's part, the circuit evaluator. Evaluating multiple records on the same encrypted circuit should be prevented: the client would learn a lot of information about the records. For every record evaluation, a new encryption of the circuit has to be constructed and transmitted. To save network resources, the server can publish the encoding of his input bits (i.e. the records) on CD-ROM without jeopardizing the secrecy of the records: in fact it's just a CD-ROM filled with random bits. The circuits the server constructs afterwards to evaluate these records must of course use the same mappings for his input bits as used on the CD-ROM.

The encoding of the client's query, which is her secret input, remains the same for all records - there's no reason to change it, any record looks the same to her. The server can choose to construct the different instances of the circuit for evaluation of the same query with identical mappings for the client's inputs. The advantage of this technique is that the $\left(\begin{array}{l}2 \\ 1\end{array}\right)-\mathrm{OT}^{k}$ only has to be executed once instead of once for every record.

If we use a symmetric 64-bit block cipher as encryption function, we can estimate the data complexity of the entire protocol as follows. Each encoding of a record takes $8 i_{s}$ bytes on CD-ROM. An encrypted NOTgate takes 32 bytes and an AND-gate takes 64 bytes, so on the average an encrypted gate takes 48 bytes, which means a scrambled (universal) circuit takes about $48 C_{u}$ bytes for each record. Using a standard implementation, the oblivious transfers take an additional $384 i_{c u}$ bytes (independent of the number of records) to be sent over the network.

\subsection{USING PROBABILISTIC ENCRYPTION}

Another technique to compute with encrypted data is based on homomorphic probabilistic encryption. An encryption technique is probabilistic if the same cleartext can encrypt to many different ciphertexts. To work with encrypted bits, probabilistic encryption is essential, otherwise only two ciphertexts (the encryption of a zero and the encryption of a one) would be possible, and cryptanalysis would be fairly simple. An encryption technique is homomorphic if it satisfies equations of the form $E(x$ op $y)=E(x) \quad \mathbf{o p}^{\prime} \quad E(y)$ for some operations op and $\mathbf{o p}^{\prime}$. A homomorphic encryption scheme allows operations to be performed on encrypted data, and hence can be used for secure circuit evaluation.

Abadi and Feigenbaum present a protocol for two-player secure circuit evaluation using a homomorphic probabilistic encryption scheme based on the Quadratic Residuosity Assumption (QRA) in [1]. This protocol 
allows $A$ who has a secret function $f$ and $B$ who has secret data $x$ to calculate $f(x)$ without jeopardizing their secrets. More precisely, the protocol allows $A$ to hide her circuit unconditionally except for the number of AND-gates, while $B$ hides his data under the QRA.

Let $k$ be the product of two primes $p$ and $q$, each congruent to $3 \bmod 4$. An integer $a \in Z_{k}^{*}[+1]$ - the integers relatively prime to $k$ with Jacobi symbol $1-$ is a quadratic residue $\bmod k$ if there exists an $x \in Z_{k}^{*}[+1]$ such that $a=x^{2} \bmod k$. The QRA states that determining if an integer $a$ is a quadratic residue mod $k$ is a hard problem if the factorization of $k$ is unknown but is easy to solve if $p$ and $q$ are given.

If we encrypt a zero by a quadratic residue and a one by a quadratic nonresidue $\bmod k$, we can define the encryption of a bit $b$ as $E_{k}(b)=$ $(-1)^{b} \cdot r^{2} \bmod k$ with $r \in_{R} Z_{k}^{*}[+1]$ chosen at random. This probabilistic encryption scheme has two homomorphic properties that will come in very handy in the protocol:

$$
\begin{aligned}
E_{k}(\bar{b}) & =(-1) \cdot E_{k}(b) \bmod k \\
E_{k}\left(b_{1} \oplus b_{2}\right) & =E_{k}\left(b_{1}\right) \cdot E_{k}\left(b_{2}\right) \bmod k
\end{aligned}
$$

$B$ starts the protocol by choosing $p$ and $q$ and multiplying them to produce $k . B$ sends $k$ and the encryption of his data $E_{k}\left(x_{1}\right), \ldots, E_{k}\left(x_{n}\right)$ to $A$. $B$ keeps the factorization of $k$ secret. $A$ then starts evaluating her secret circuit. If she has to evaluate a NOT gate with input $E_{k}(b)$, she simply calculates $-E_{k}(b) \bmod k$. An XOR with inputs $E_{k}\left(b_{1}\right)$ and $E_{k}\left(b_{2}\right)$ is also easy to evaluate: $A$ just takes $E_{k}\left(b_{1}\right) \cdot E_{k}\left(b_{2}\right) \bmod k$ as the output of the gate. To evaluate the AND of inputs $E_{k}\left(b_{1}\right)$ and $E_{k}\left(b_{2}\right)$, she needs $B$ 's help. $A$ chooses two bits $c_{1}$ and $c_{2}$ at random and sends $E_{k}\left(b_{1} \oplus c_{1}\right)$ and $E_{k}\left(b_{2} \oplus c_{2}\right)$ to $B$. $B$ decrypts the bits $A$ just sent him as $d_{1}$ and $d_{2}$ (he can do so because he knows $p$ and $q$ ) and sends the tuple $<E_{k}\left(d_{1} \wedge d_{2}\right), E_{k}\left(d_{1} \wedge \overline{d_{2}}\right), E_{k}\left(\overline{d_{1}} \wedge d_{2}\right), E_{k}\left(\overline{d_{1}} \wedge \overline{d_{2}}\right)>$ to $A$. $A$ takes the first element of this tuple as the output of the AND gate if she chose $c_{1}=c_{2}=0$, the second if she chose $c_{1}=0$ and $c_{2}=1$, the third if she chose $c_{1}=1$ and $c_{2}=0$ and the last one if she chose $c_{1}=c_{2}=1$. Proceeding this way from gate to gate, $A$ ends with the encrypted result $E_{k}(f(x))$ and sends it for decryption to $B$.

The evaluation of an AND-gate requires six encrypted bits to be sent over the network. We can reduce this number to three by applying a little trick Franklin and Haber used in [5]. Just like the original protocol, $A$ chooses random bits $c_{1}$ and $c_{2}$ and sends $E_{k}\left(b_{1} \oplus c_{1}\right)$ and $E_{k}\left(b_{2} \oplus c_{2}\right)$ to $B$. $B$ again decrypts these bits as $d_{1}$ and $d_{2}$ but sends only $E_{k}\left(d_{1} \wedge d_{2}\right)$ back to $A$. Now, it is easy to prove that

$$
d_{1} \wedge d_{2}=\left(b_{1} \wedge b_{2}\right) \oplus\left(b_{1} \wedge c_{2}\right) \oplus\left(b_{2} \wedge c_{1}\right) \oplus\left(c_{1} \wedge c_{2}\right)
$$


or, applying the homomorphism between $\oplus$ and multiplication $\bmod k$,

$$
E_{k}\left(b_{1} \wedge b_{2}\right)=E_{k}\left(d_{1} \wedge d_{2}\right) \cdot E_{k}\left(b_{1} \wedge c_{2}\right) \cdot E_{k}\left(b_{2} \wedge c_{1}\right) \cdot E_{k}\left(c_{1} \wedge c_{2}\right)
$$

$A$ just got $E_{k}\left(d_{1} \wedge d_{2}\right)$ from $B$ and she can easily make an encryption of $c_{1} \wedge c_{2}$. Computing $E_{k}\left(b_{1} \wedge c_{2}\right)$ is a little more subtle. If she chose $c_{2}$ to be 0 , she can take any encryption of a zero to be that factor. If she chose $c_{2}$ to be 1 , she uses $E_{k}\left(b_{1}\right)$ itself. The last factor, $E_{k}\left(b_{2} \wedge c_{1}\right)$, is calculated in an analogous way.

This protocol can do a great job in a Secret Query Database. Unlike the previous protocol, we don't need a universal circuit if we let the client play the role of $A$ and let the secret function be her query $q$ itself. The server plays the role of $B$, supplying encrypted records as his secret data.

The length of $k$ is an important security parameter: if a client is able to compute the factorization of $k$, she can decrypt the record. At this moment, it is possible to factor 512-bit numbers if you are willing to spend a few thousands of dollars and wait a few months. The plaintext of the entire database could be worth this effort, but one single record probably isn't. If we use a new 512-bit $k$ for every record, an attacker has to factor a 512-bit number for every record he wants. For most databases it will be cheaper simply to buy the records from the server.

In spite of the relatively small key length, the encryption of a record still causes a huge data blow: it's size is multiplied by a factor of 512 . Our records of $i_{s} / 8$ bytes take $64 i_{s}$ bytes in encrypted form. Of course, it is intolerable to send this kind of data over a network. Yet the factors of $k$ are not revealed during the protocol, allowing the data to be reused in multiple evaluations. Again, the server can publish encrypted records and the public key $k$ used for the encryption on CD-ROM to drastically reduce network load.

As we stated above, the evaluation of an AND-gate needs three encrypted bits to be sent over the network. If the client's circuit consists of $C$ gates, half of which are NOT-gates or XOR-gates that require no interaction and half of which are AND-gates that do require interaction, on the average $96 \mathrm{C}$ bytes are to be sent over the network for each record evaluation.

\subsection{AUTONOMOUS PROTOCOLS}

The protocols discussed in the two previous subsections require more communication rounds than strictly necessary. Although it is impossible (as discussed in section 2) to construct a completely non-interactive solution, it turns out to be possible to construct solutions using only one communication round: the client sends (in one message) an encrypted 
function $f$, and it receives from the server an encrypted result $f(x)$ in such a way that $f$ remains private to the client and $x$ remains private to the server. A protocol that achieves this result in only one communication round is called an autonomous protocol.

Two kinds of autonomous protocols have been proposed in the literature. The first kind, introduced by Sander and Tschudin ([8]), allows for a fairly efficient evaluation of polynomials in a ring of integers modulo $n$ using a homomorphic encryption scheme. However, it is not clear if this technique can be extended to general boolean circuits, and hence it does not seem applicable to the Secret Query Database.

The second kind, introduced by Loureiro and Molva ([7]), uses a public key encryption system based on Goppa codes, and allows for the evaluation of functions describable by a matrix multiplication. Loureiro and Molva also show how any boolean circuit evaluation can be done by a matrix multiplication. However, the representation of a boolean circuit requires a huge matrix, which renders the technique hopelessly inefficient for application in the Secret Query Database.

Hence, for the current state of the art of autonomous protocols, it seems that these protocols cannot compete with the protocols discussed in the sections above.

\section{ASSESSMENT AND CONCLUSION}

We compare the two most promising solutions of all the solutions discussed in section 3. A distinction is made between (1) the amount of data that can be published once and for all (e.g. on a CD-ROM), (2) the amount of data that must be exchanged once per query (but can be reused when evaluating the query on successive records) and (3) the amount of data that must be exchanged for each individual query evaluation. We call the first kind of data $C D-R O M$ data, the second kind query setup data and the third kind query evaluation data. We consider the (realistic) numerical values discussed in section 2, and assume a database of $n_{r}=1024$ records. Table 1 summarizes the communication overhead.

For the oblivious transfer based solution, the total network communication overhead to query the entire database becomes $375 \mathrm{~K}+1024 \cdot 190 \mathrm{~K}$ or about 190 megabytes. For the probabilistic encryption based solution, this total overhead becomes $1024 \cdot 94 \mathrm{~K}$ or 94 megabytes. Although these numbers are indeed rather high, it is important to note that they can be reduced by either lowering the required security level, or by reducing query complexity. In other words, the overhead can be traded off versus security or functionality. 


\begin{tabular}{llll}
\hline & CD-ROM & query setup & query evaluation \\
\hline $\begin{array}{l}\text { OT-based } \\
\text { solution }\end{array}$ & $8 n_{r} i_{s}$ bytes & $384 i_{c u}$ bytes & $48 C_{u}$ bytes $/$ record \\
& $=32$ Mbytes & $=375 \mathrm{Kbytes}$ & $=190 \mathrm{Kbytes} / \mathrm{record}$ \\
$\begin{array}{l}\text { PE-based } \\
\text { solution }\end{array}$ & $\begin{array}{l}64 n_{r} i_{s} \text { bytes } \\
=250 \mathrm{Mbytes}\end{array}$ & 0 bytes & $\begin{array}{l}96 \mathrm{~b} \text { bytes } / \mathrm{record} \\
=94 \mathrm{Kbytes} / \mathrm{record}\end{array}$ \\
\hline
\end{tabular}

Table 1 Summary of communication overhead

Hence, we believe it is fair to state that secure distributed computing technology is ready to be considered for practical applications.

\section{References}

[1] M. Abadi and J. Feigenbaum, "Secure circuit evaluation, a protocol based on hiding information from an oracle," Journal of Cryptology, 2(1), p. 1-12, 1990

[2] B. Chor, O. Goldreich, E. Kushilevitz and M. Sudan, "Private information retrieval," Proc. of 36th IEEE Conference on the Foundations of Computer Science (FOCS), p. 41-50, 1995

[3] D.E. Denning and J. Schlörer, "A Fast Procedure for Finding a Tracker in a Statistical Database," ACM Transactions on Database Systems, 5, 1, p. 88-102, 1980.

[4] M. Franklin, "Complexity and security of distributed protocols," Ph. D. thesis, Computer Science Department of Columbia University, New York, 1993

[5] M. Franklin and S. Haber, "Joint encryption and message-efficient secure computation," Journal of Cryptology, 9(4), p. 217-232, Autumn 1996

[6] O. Goldreich, S. Micali and A. Wigderson, "How to play any mental game," Proc. of 19th ACM Symposium on Theory of Computing (STOC), p. 218-229, 1987

[7] S. Loureiro and R. Molva, "Privacy for Mobile Code", Proceedings of the workshop on Distributed Object Security, OOPSLA '99, p. 3742.

[8] T. Sander and C. Tschudin, "On software protection via function hiding", Proceedings of the second workshop on Information Hiding, Portland, Oregon, USA, April 1998. 